\title{
Repeated Intravenous Thrombolysis in Patients with Recurrent Ischemic Stroke in the Vertebrobasilar Territory
}

\author{
David Černík ${ }^{a} \quad$ Dušan Ospalík ${ }^{a} \quad$ Daniel Šaňák ${ }^{b} \quad$ Filip Cihlářc $^{c}$ \\ aDepartment of Neurology, Comprehensive Stroke Center, Masaryk Hospital Ústí \\ nad Labem, KZ a.s., Ústí nad Labem, Czech Republic; ${ }^{b}$ Department of Neurology, \\ Comprehensive Stroke Center, Palacký University Medical, School and Hospital, Olomouc, \\ Czech Republic; 'Department of Radiology, Masaryk Hospital, Faculty of Health Studies, J.E. \\ Purkinje University, KZ a.s., Ústí nad Labem, Czech Republic
}

\section{Keywords}

Repeated intravenous thrombolysis · Ischemic stroke · Basilar artery occlusion · Eptifibatide

\begin{abstract}
Acute ischemic vertebrobasilar stroke (AIVBS) is usually associated with poor outcome and prognosis and in case of basilar artery occlusion (BAO) with high mortality. Intravenous thrombolysis (IVT), as a standard recanalization therapy of acute ischemic stroke (IS) within first $4.5 \mathrm{~h}$, can be administrated beyond this therapeutic time window in case of symptomatic BAO. Repeated IVT is generally contraindicated in case of early recurrent IS, despite a risk of poor outcome or death after recurrent IS. The aim was to present 2 cases of repeated IVT for recurrent AIVBS and discuss specific situations where repeated IVT may be considered. Up to now, repeated IVT has been reported only in recurrent stroke in anterior circulation.

\section{Introduction}

Acute ischemic vertebrobasilar stroke (AIVBS) has typically a poor prognosis, while ischemic stroke (IS) with the occlusion of the basilar artery (BA) is mostly fatal [1]. Intravenous thrombolysis (IVT) as a standard recanalization therapy for acute IS within the first $4.5 \mathrm{~h}$ after symptom onset may be administered beyond $4.5 \mathrm{~h}$ in case of the symptomatic BA occlusion (BAO) [2]. The reason for an extension of the therapeutic time window is the presence of a very high probability of death or a poor outcome after stroke [1]. Despite the fact that the early recurrence of IS, especially in the BA territory, can be fatal for the patient, the repeated administration of IVT remains 
Černík et al.: Repeated IVT for Recurrent Ischemic Stroke in the Vertebrobasilar Territory

generally contraindicated [2]. The aim of this contribution is to highlight specific situations where the repeated administration of IVT could be considered in case of recurrent IS in the BA territory.

\section{Case 1}

A 74-year-old male underwent recurrent IS in the carotid territory in 2012, and according to CT angiography (CTA), atherosclerotic changes were present, including asymptomatic stenosis of the BA. He was administered a combination of $100 \mathrm{mg}$ of acetylsalicylic acid (ASA) and $75 \mathrm{mg}$ of clopidogrel daily as a prevention. He was able to walk independently (a broader basis) without any aid (zero points in the National Institutes of Health Stroke Scale [NIHSS] and 1 point in the modified Rankin scale [mRS]).

In August 2015, he was admitted for acute IS presented with dysarthria, central palsy of the right facial nerve, and ataxia (NIHSS score: 6 points). No signs of acute ischemia were observed on the admission noncontrast brain CT, and an occlusion of the BA was detected at the site of known stenosis on the following CTA. Given the unfavorable anatomy, the interventional radiologist did not indicate endovascular intervention, and thus IVT was administered (80 mg Actilyse ${ }^{\circledR}$; door to needle time [DNT], $35 \mathrm{~min}$ ) with subsequent regression of admission deficit to residual right-sided ataxia (NIHSS 1 and mRS 1). On the control CT scan after $24 \mathrm{~h}$, no ischemic changes were observed. CTA was not performed, as it would only represent a radiation burden for the patient without impact on the further therapeutic procedure. In the secondary prevention, combined antiplatelet and anticoagulant therapy (100 mg ASA and LMWH [Fraxiparine ${ }^{\circledR} 0.4 \mathrm{~mL}$ twice daily]) and statin were administered and tiapridal intermittently during unrest. Fifty hours after the administration of IVT, the sudden development of quadriparesis with impaired consciousness occurred (NIHSS 24). A native brain CT did not show new acute ischemic changes, and on the following CTA, occlusion of the BA remained (we assume progressive chronic occlusion with distal embolization), and newly, the occlusion of the right vertebral artery (VA) occurred. Endovascular intervention was not performed again due to technical reasons - challenging anatomy with severe tortuosity of the subclavian artery and the VA and possible chronic occlusion of the BA. Favorable result after previous IVT and generally poor results of the endovascular intervention in case of the basilar atherosclerotic stenosis/occlusion were the additional arguments for our treatment decision. Thus, repeated IVT was administered (80 mg Actilyse ${ }^{\circledR}$, DNT $44 \mathrm{~min}$ ) with a significant regression of neurological deficit into moderate bilateral limiting independent walking only indoors (NIHSS 2 and mRS 3). Due to the failure of the previous combined antiplatelet and anticoagulation therapy, continuous administration of eptifibatide (Integrilin ${ }^{\circledR}$ ) was initiated. The control CTA after 24 $\mathrm{h}$ showed recanalization of the right VA and persisting occlusion of the BA. No new ischemic changes and no hemorrhage after IVT were detected. On the following MRI, small ischemic lesions in both cerebellar hemispheres were present, without the finding of acute ischemia in the brainstem. The patient was subsequently treated with a combination of anticoagulation therapy (LMWH; Fraxiparine ${ }^{\circledR} 0.8 \mathrm{~mL}$ twice a day) and dual antiplatelet therapy (Aggrenox ${ }^{\circledR}$ ) and transferred into a rehabilitation facility (NIHSS 2 and mRS 3). After 5 months, he was capable of basic self-care and independent walking using a low walker (NIHSS 0 and mRS 2).

\section{Case 2}

A 52-year-old male suffered IS with a residual mild right upper limb paresis (NIHSS 1 and mRS 1) in 2013. A megadolichobasilaris (within dolichoectasia of the intracranial arteries) was detected on CTA. He was administered $100 \mathrm{mg}$ of aspirin per day. In November 2015,

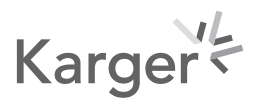


he was admitted for acute onset of left-sided central hemiparesis with impaired sensation with central paresis of the right facial nerve (NIHSS 5). On the admission noncontrast brain $\mathrm{CT}$, no acute ischemic changes were observed, and insignificant atherosclerotic plaque in the BA was detected on the CTA. After the administration of IVT ( $60 \mathrm{mg}$ Actilyse ${ }^{\circledR}$, DNT $44 \mathrm{~min}$ ), residual slight movement impairment of the right limb was present (NIHSS 2 and mRS 1). No signs of ischemic changes were present on the control MRI. The patient was switched from ASA to clopidogrel (recurrence of IS on ASA and the increased risk of cerebral hemorrhage resulting from dolichoectasia of the cerebral arteries [3]) in combination with statin (rosuvastatin $20 \mathrm{mg}$ ). After the patient's discharge to home, a radiologist reevaluated the MRI finding of a plaque in the BA as a mural thrombosis without evolution over time. Thus, the patient returned to the hospital for an initiation of anticoagulation therapy 11 days after the administration of IVT. Four hours after the patient's return to the hospital, he developed suddenly dysarthria with palsy of the right facial nerve and right-sided hemiparesis (NIHSS 8).

On the immediately performed CT, no signs of acute ischemia were present, and embolization of the thrombus in the BA was suspected. Repeated IVT was administered (60 $\mathrm{mg}$ Actilyse ${ }^{\circledR}$, DNT $33 \mathrm{~min}$ ), with a prompt recovery (NIHSS 2 and mRS 1). In secondary prevention, the patient was switched to oral anticoagulation therapy with warfarin.

In March 2016, a week after dose reduction of warfarin with following decrease of INR from 2.5 to 1.3, the patient suffered from a recurrent IS (right-sided hemiparesis with dysarthria, NIHSS 5). On the admission CTA, thrombus in the BA was not present. IVT was administered (60 mg Actilyse ${ }^{\circledR}$, DNT $43 \mathrm{~min}$ ), but the deficit fluctuated. After the control CT, eptifibatide was administered intravenously for the next $24 \mathrm{~h}$. Subsequent partial regression of deficit to moderate paresis of the right upper extremity (NIHSS 5 and mRS 2) occurred.

\section{Discussion}

Currently, IVT remains still contraindicated for patients with a history of previous IS in the last 3 months with severe residual clinical or radiological findings [4]. We performed repeated IVT in both presented cases regarding the fact that the ischemia in the VB territory may be fatal or associated with poor outcome in patients with initially severe deficit. The BAO represents the specific situation in the treatment of IS in posterior circulation, where IVT may be administered beyond standard $4.5 \mathrm{~h}$ based on extremely high probability of the patient's death. Moreover, the later recanalization decreases the chance for clinical improvement and survival, and even in the posterior circulation, shortening onset-to-treatment time has an effect on good clinical outcome [5].

Dorńák et al. [1] have documented a severe prognosis of patients with stroke in the VB territory in a group of 51 patients; only $30 \%$ of them achieved good clinical outcome (mRS $0-2$ ) at 3 months after the stroke. The recanalization rate after IVT does not exceed 15-30\% in the occlusion of large cerebral arteries [6-8]. Thus, endovascular treatment is recommended as a standard treatment option [9-11] for IS in anterior circulation. In case of BAO, endovascular treatment should be also considered, even beyond the recommended 6-h time window for stroke in anterior circulation as life-threatening therapy. In our case, the endovascular therapy of BAO was not performed due to described anatomical and technical limitations.

Early repeated administration of IVT has been reported only in the form of case studies. Topakian et al. [12] reported the use of IVT repeatedly up to $90 \mathrm{~h}$ in the case of recurrent IS in the anterior circulation, and recently, Bouchal et al. [13] reported recurrent IS in the anterior circulation. Wu et al. [14] reported 57 cases with the time interval between repeated administration of IVT $<14$ days only in 20 of them (in 10 of them within $48 \mathrm{~h}$ ).

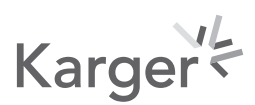


The risk of cerebral hemorrhagic complications after IVT was reported significantly lower in the VB territory compared to anterior circulation $[15,16]$, and it may be suggested that also risk of hemorrhagic complications after repeated IVT could be lower in IS in the VB territory. We used eptifibatide (Integrilin ${ }^{\circledR}$, antagonist IIb/IIIa receptor blocking the final common pathway of platelet aggregation) after the failure of conventional therapy. According to the previous reports, the co-administration of IVT and eptifibatide did not lead to the increase of complications and showed certain clinical benefit [17-19].

Despite the positive results of repeated, uncomplicated administration of IVT in our patients, limitations related to presentation of the 2 isolated cases from 1 center must be considered. In conclusion, repeated early administration of IVT in both presented cases led to significant clinical improvement. In the first presented patient, IVT was probably lifethreatening. None of the both patients had complications. The use of eptifibatide during the failure of a conventional antiplatelet and anticoagulant therapy to reduce the risk of new thrombotic complications in the first hours after the administration of IVT was not associated with complications. The early recurrence of IS in the VB territory may be fatal, and thus repeated administration of IVT might be considered in individual cases as a life-threatening treatment.

\section{Statement of Ethics}

Ethical approval was not necessary for the preparation of this article. Written informed consent was obtained from patients for publication.

\section{Conflict of Interest Statement}

The authors have no conflicts of interest to declare.

\section{Funding Sources}

This work was partially supported by the grant Krajská zdravotní a.s. IGA-KZ-2019$1-3$.

\section{Author Contributions}

David Černík was involved in manuscript writing - case 1. Dušan Ospalík was involved in manuscript writing - case 2. Daniel Šaňák critically revised the whole manuscript content. Filip Cihlář was involved in manuscript writing.

\section{Data Availability Statement}

All data generated or analyzed during this study are included in this article and/or its online suppl. material files (for all online suppl. material, see www.karger.com/ doi/10.1159/000518193). Further enquiries can be directed to the corresponding author. 
Černík et al.: Repeated IVT for Recurrent Ischemic Stroke in the Vertebrobasilar Territory

\section{References}

1 Dorňák T, Herzig R, Školoudík D, Sanák D, Kuliha M, Roubec M, et al. Outcome predictors in acute basilar artery occlusion. Can J NeurolSci. 2014;41:368-74.

2 The European Stroke Organisation (ESO) Executive Committee a the ESO Writing Committee. Guidelines for management of ischaemic stroke. 2008 Update 2009, Available from: http://www.eso-stroke.org/eso-stroke/ education/guidelines.html Accessed 2021 Mar 20.

3 Pico F, Labreuche J, Amarenco P. Pathophysiology, presentation, prognosis, and management of intracranial arterial dolichoectasia. Lancet Neurol. 2015;14:833-45.

4 Neumann J, Tomek A, Školoudík D, Škoda O, Mikulík R, Herzig R, et al. Guidelines for intravenous thrombolysis in acute ischemic stroke: version 2014. Cesk Slov Neurol N. 2014;77/110(3):381-55.

5 Huang Q, Song HQ, Ma QF, Song XW, Wu J. Effects of time delays on the therapeutic outcomes of intravenous thrombolysis for acute ischemic stroke in the posterior circulation: an observational study. Brain Behav. 2019 Feb;9(2):e01189.

6 Muchada M, Rodriguez-luna D, Pagola J, Flores A, Sanjuan E, Meler P, et al. Impact of time to treatment on tissue-type plasminogen activator-induced recanalization in acute ischemic stroke. Stroke. 2014;45(9): 2734-8.

7 Alexandrov AV, Molina CA, Grotta JC, Garami Z, Ford SR, Alvarez-Sabin J, et al. Ultrasound-enhanced systemic thrombolysis for acute ischemic stroke. N Engl J Med. 2004;351(21):2170-8.

8 Molina CA, Ribo M, Rubiera M, Montaner J, Santamarina E, Delgado-Mederos R, et al. Microbubble administration accelerates clot lysis during continuous 2-MHz ultrasound monitoring in stroke patients treated with intravenous tissue plasminogen activator. Stroke. 2006;37:425-9.

9 Šaňák D, Mikulík R, Tomek A, Bar M, Herzig R, Neumann J, et al. Guidelines for mechanical thrombectomy in acute ischemic stroke: version 2019. Cesk Slov Neurol N. 2019;82/115(6):700-5.

10 Saver JL, Goyal M, Bonafe A, Diener HC, Levy EI, Pereira VM, et al. Stent-retriever thrombectomy after intravenous t-PA vs. t-PA alone in stroke. N Engl J Med. 2015;372:2285-95.

11 Berkhemer OA, Fransen PS, Beumer D, van den Berg LA, Lingsma HF, Yoo AJ, et al. A randomized trial of intraarterial treatment for acute ischemic stroke. N Engl J Med. 2015 Jan 1;372(1):11-20.

12 Topakian R, Gruber F, Fellner FA, Haring HP, Aichner FT. Thrombolysis beyond the guidelines: two treatments in one subject within 90 hours based on a modified magnetic resonance imaging brain clock concept. Stroke. 2005;36:e162-4.

13 Bouchal S, Lamrani YA, Chtaou N, Maaroufi M, Belahsen F. Repeated intravenous thrombolysis in early recurrent stroke secondary to carotid web: case report. Radiol Case Rep. 2021;16(4):843-6.

$14 \mathrm{Wu}$ J, Huang $\mathrm{Q}$, Ma Q. What matters in the results of repeated intravenous thrombolysis for recurrent ischemic stroke? EurNeurol. 2016;75:150-4.

15 Dorňák T, Král M, Hazlinger M, Herzig R, Veverka T, Buřval S, et al. Posterior vs.anterior circulation infarction: demography, outcomes and frequency of hemorrhage after thrombolysis. Int J Stroke. 2015;10:1224-8.

16 Tong X, Liao X, Pan Y, Cao Y, Wang C, Liu L, et al. Intravenous thrombolysis is more safe and effective for posterior circulation stroke: data from the thrombolysis implementation and monitor of acute ischemic stroke in China (TIMS-China). Medicine. 2016;95(24):e3848.

17 Pancioli AM, Adeoye O, Schmit PA, Khoury J, Levine SR, Tomsick TA, et al. Combined approach to lysis utilizing eptifibatide and recombinant tissue plasminogen activator in acute ischemic stroke-enhanced regimen stroke trial. Stroke. 2013;44(9):2381-7.

18 Adeoye O, Sucharew H, Khoury J, Tomsick T, Khatri P, Palesch Y, et al. Recombinant tissue-type plasminogen activator plus eptifibatide versus recombinant tissue-type plasminogen activator alone in acute ischemic stroke: propensity score-matched post hoc analysis. Stroke. 2015;46:461-4.

19 Dubey D, Banerjee C, Sawhney A, Sharma A, Alberts MJ. Combination therapy of intravenous glycoprotein IIB/ IIIA inhibitors and tissue plasminogen activator for acute ischemic stroke. Neurol India. 2014;62:631-4. 